Heugten, C.M. van, Dekker, J., Deelman, B.G., Stehmann-Saris, J.C., Kinebanian, A. Rehabilitation of stroke patients with apraxia: the role of additional cognitive and motor impairments.

Disability and Rehabilitation: 22, 2000, nr. 12, p. 547-554

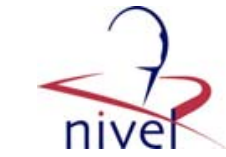

\begin{tabular}{|l|l|}
\hline Postprint Version & 1.0 \\
\hline Journal website & http://www.informaworld.com/smpp/title $\sim$ content=t713723807 \\
Pubmed link & $\begin{array}{l}\text { http://www.ncbi.nlm.nih.gov/entrez/query.fcgi?cmd=Retrieve\&db=pubmed\&dop } \\
\text { t=Abstract\&list_uids=11005744\&query_hl=75\&itool=pubmed_docsum }\end{array}$ \\
\hline DOI & \multicolumn{2}{|l|}{}
\end{tabular}

\title{
Rehabilitation of stroke patients with apraxia: the role of additional cognitive and motor impairments
}

\author{
C. M. VAN HEUGTEN ${ }^{1,2^{*}}$, J. DEKKER ${ }^{1,3}$, B. G. DEELMAN ${ }^{4}$, J. C. STEHMANN-SARIS $^{5}$ AND A. \\ KINEBANIAN $^{5}$
}

1Netherlands Institute of Primary Health Care (NIVEL), P.O. Box 1568, 3500 BN Utrecht, The Netherlands

2Institute for Rehabilitation Research, Hoensbroek, The Netherlands

3Vrije Universiteit, Amsterdam, The Netherlands

4Department of Neuropsychology, University of Groningen, The Netherlands

5Polytechnic of Amsterdam, School for Occupational Therapy, Amsterdam, The Netherlands

*Author for correspondence; e-mail: c.vanheugten@irv.nl

\begin{abstract}
Purpose: The present study investigated which additional cognitive and motor impairments were present in stroke patients with apraxia and which of these factors influenced the effects of treatment.

Method: A group of 33 patients with apraxia were treated according to the guidelines of a therapy programme based on teaching patients strategies to compensate for the presence of apraxia. Patients were treated at occupational therapy departments in general hospitals, rehabilitation centres and nursing homes. The outcome of the strategy training was studied in a pre-post test design; measurements were conducted at baseline and after 12 weeks of therapy. The pretreatment scores of the patients with apraxia were compared to normscores and scores of a control group of patients without apraxia $(n=36)$ to investigate which impairments are present. The following variables were analysed in order to determine which factors influence outcome: additional neuropsychological deficits (comprehension of language, cognitive impairments due to dementia, neglect and short term memory), level of motor functioning, severity of apraxia and performance on activities of daily living (ADL), and some relevant patient characteristics (gender, age, type of stroke, time since stroke, and location of treatment).

Results: The results showed that the presence of apraxia is associated with the presence of additional cognitive and motor impairments. The successful outcome of strategy training was not negatively influenced by cognitive comorbidity. The outcome seemed to be more prominent in patients who were more severely impaired at the start of rehabilitation in terms of the degree of motor impairments, the severity of apraxia and the initial ADL dependence. The ADL observations, however, displayed a ceiling effect, which was taken into account in discussing the results. Demographic variables, especially age, did not predict the outcome of treatment.
\end{abstract}

Conclusions: We suggest that the effect of this training is stronger in more severely disabled patients. However, neither the presence of additional cognitive impairments nor 
Heugten, C.M. van, Dekker, J., Deelman, B.G., Stehmann-Saris, J.C., Kinebanian, A.

Rehabilitation of stroke patients with apraxia: the role of additional cognitive and

motor impairments.

Disability and Rehabilitation: 22, 2000, nr. 12, p. 547-554

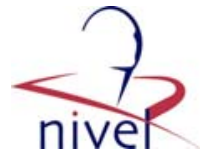

the severity of motor problems nor old age should be an indication for refraining from treating apraxia.

\section{INTRODUCTION}

Identification of patients who will benefit more than others from a specific treatment is important for a number of reasons. Prognosis of the outcome is essential to the caregivers : realistic rehabilitation goals can be formulated ${ }^{1}$ and health care services can be allocated in the most efficient way when factors affecting outcome are identified. The caregivers and, perhaps even more importantly, the patients themselves as well as their relatives should be informed adequately about the expected outcome. Finally, concerning research in rehabilitation, knowledge of prognosis facilitates stratification of stroke patients into different prognostic groups for clinical trials. ${ }^{2}$ In designing randomized clinical trials, this makes it possible to create more homogeneous groups of patients. Most prognostic studies do not differentiate between spontaneous recovery and the effects of a specific treatment ; instead, recovery after stroke in general is examined. In a recent review, many studies on the prognosis of stroke patients were analysed and variables capable of predicting functional outcome after stroke were identified on the basis of those studies that met sound methodological principles of prognostic research. ${ }^{3}$ Kwakkel et al. identified the following negative predictors for functional recovery after stroke : old age, previous stroke, urinary incontinence, loss of consciousness at onset, disorientation in time and place, severity of paralysis, poor sitting balance, bad admission ADL score, low level of social support, and metabolic rate of glucose outside the infarct area in hypertensive patients.

Kwakkel et al. studied stroke patients in general ; the present study focused on stroke patients with apraxia in particular. Basso and colleagues ${ }^{4}$ investigated the recovery from ideomotor apraxia (IMA) in acute stroke patients and assessed whether variables capable of predicting the evolution of IMA could be identified. They found that recovery is related to the site of the lesion : patients with anterior lesions appeared to have a better chance of recovery. Improvement was not related to age, education, sex, type of aphasia, and the initial severity or the size of the lesion. A study by Sundet et al. ${ }^{5}$ was designed to investigate how apraxia (among other variables) present at the start of rehabilitation, may predict the level of ADL dependency after discharge. These authors found that variables associated with apraxia at the start of rehabilitation correlated significantly with the level of help needed for managing alone at home after discharge : apraxia on admission indicated a higher dependency on aids and other persons in order to manage alone. From these results, however, the effect of rehabilitation or spontaneous recovery cannot be differentiated.

In the present paper the influence of several variables on treatment was explored : baseline performance (baseline degree of apraxia and ADL performance), and a number of relevant patient characteristics (age, gender, type of stroke, time since stroke and location of treatment). Perhaps more importantly, in the present paper the role of additional cognitive and motor impairments was investigated. First, it was examined whether the apractic patients in the study indeed suffered from other impairments besides apraxia. This was done by comparing the pretreatment scores of the patients with apraxia with normscores and with scores of a control group of stroke patients without apraxia. Next, an attempt was made to identify those variables that influenced the outcome of treatment, as measured with standardized ADL observations. We expected that the presence of additional neuropsychological and motor deficits would have a negative influence on functional outcome. Obviously, these deficits can have a negative influence on independent functioning ; therefore it is important to determine whether the outcome of treatment specifically aimed at apraxia will be worse when patients suffer from comorbidity. Patients having apraxia without (or with less) cognitive and motor comorbidity were expected to benefit more from treatment.

\section{METHODS}

\section{Selection of patients}

Therapists at occupational therapy departments in three general hospitals, eight rehabilitation centres, and five nursing homes in the Netherlands selected the subjects. Patients were eligible if they met the following inclusion criteria : patients diagnosed as having had a stroke in the left hemisphere, and 
Heugten, C.M. van, Dekker, J., Deelman, B.G., Stehmann-Saris, J.C., Kinebanian, A.

Rehabilitation of stroke patients with apraxia: the role of additional cognitive and

motor impairments.

Disability and Rehabilitation: 22, 2000, nr. 12, p. 547-554

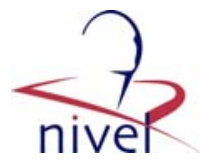

having apraxia. A left hemisphere stroke was diagnosed when acute clinical symptoms of a focal dysfunction of the left hemisphere were present at least during 24 hours and probably had a vascular origin only. ${ }^{6,7}$ Apraxia was diagnosed when the patient was fully or partly unable to carry out purposeful activities, this not being due to primary motor or sensory impairments, nor to deficits of comprehension, memory, or motivation. ${ }^{8}$ Exclusion criteria were : age younger than 25 years or older than 95 years; no working knowledge of the Dutch language; and a set of premorbid or present pathologies : psychiatric or psychogeriatric history, addiction to alcohol, medical or other drugs, personality, intellectual or learning disorders, temporary loss of consciousness, or other injuries of the central nervous system.

A group of stroke patients without apraxia was selected on the basis of the same inclusion- and exclusion criteria as the first group, but they were not diagnosed as having apraxia. All subjects in both groups entering the study gave their informed consent. Both groups of patients were recruited by the occupational therapists during a period of 17 months and all patients meeting the criteria were intended to be included consecutively. Due to organizational problems (absence of a therapist, time schedules, etc.) or patients already participating in other research projects, not all suitable patients were actually included ; the group of patients with apraxia consisted of 33 subjects and the group of patients without apraxia consisted of 36 subjects.

\section{Treatment}

The patient eligible for the study took part in an assessment procedure and were subsequently treated according to the guidelines of a therapy programme for a period of 12 weeks. Treatment was aimed at improving the performance of the apractic patients by teaching them strategies. The potential benefit of this is that it enables them to function more independently, despite the persisting presence of apraxia. Strategy training concentrates on teaching the patient ways to compensate for the impairment. The number of treatments per week was determined by the therapists, and varied between three to five sessions per week. During treatment activities were trained that were relevant for the patients to (re)learn. The focus of the programme was on disabilities resulting from apraxia, and having an influence on the patient' s functioning in daily life. Every two weeks an activity was chosen. The decision about the activity to be trained was made together with the patient. After this two week period a new treatment goal was chosen, based upon the improvement in the patient' s functioning.

The specific interventions administered during treatment corresponded with the specific problems that were assessed during ADL-observations. ${ }^{9}$ ADL activities were conceptualized as being composed of three aspects : phases of initiation, execution and control. The proper plan of action as well as the correct objects have to be selected (initiation of an activity), followed by adequate performance of the plan (executing the activity), which has to be evaluated in terms of the performance and the result (controlling and if necessary correcting the activity). By assessing these different aspects of the activity, the nature of the deficit can be identified and plans for treatment can be formulated accordingly. When a patient predominantly showed problems with initiating an activity, emphasis during treatment was placed upon instructions. Specific assistance was given when the execution of an activity caused problems. Finally, the therapist offered feedback when the patient did not detect or correct performance errors. The specific interventions in the form of instructions, assistance, and feedback had been presented to the occupational therapist in a protocol.

\section{Study design and outcome measures}

The outcome of the therapy programme was studied using a pre-post test design. The following measurements were performed at baseline (pre test) and 12 weeks later (post test):

\section{Apraxia}

A test of apraxia, consisting of two subtests, ${ }^{10}$ based on tests of De Renzi ${ }^{11}$ was used. The first subtest was designed to evaluate the use of objects. Three sets of three objects were presented in different conditions. A total score of 54 could be reached. The second subtest involved the assessment of the ability to imitate gestures. Six gestures had to be imitated by the patient, directly upon demonstration by the researcher. For this part a score of 36 could be reached. The total score could add up to 90: a higher score denotes better performance. The internal consistency (alpha $=0.96)$ and the validity of this test were investigated; both clinimetrical properties appeared to be good. ${ }^{10}$ 
Heugten, C.M. van, Dekker, J., Deelman, B.G., Stehmann-Saris, J.C., Kinebanian, A.

Rehabilitation of stroke patients with apraxia: the role of additional cognitive and

motor impairments.

Disability and Rehabilitation: 22, 2000, nr. 12, p. 547-554

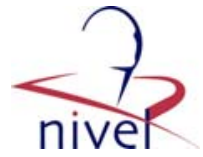

\section{Motor functioning}

A short and simple test of motor functioning was used, based on other motor functioning tests (e.g. the motricity index). ${ }^{12}$ The test consists of 8 tasks concerning the body side opposite to the brain lesion, involving trunk balance, shoulder movement, arm movement, grasp and release a cylinder, grasp and release a dice, and a test for the sensitivity of the back of the hand. For each task a score of 2 was given when the patient was able to execute the task, 1 when the patient could carry it out with some effort, and 0 when the patient was not able to execute the task. This way a maximum score of 16 could be reached. The internal consistency of this test is good (alpha $=0.94$; not published).

\section{ADL-observations}

The occupational therapist executed a set of four standardized ADL observations aiming at assessing abilities and disabilities following apraxia. The OT observed the patient executing four ADLactivities, three of which were prescribed (i.e. personal hygiene, dressing and preparing food), while the fourth activity could be chosen by the OT. The findings of these observations were scored on four different scores : a score of independence, initiation, execution and control. The four measures consisted of 4 levels ranging from `there are no observable problems, the patient performs well without help' (0), to 'the therapist has to take over the activity' (3). Subsequently, the four measures were added to arrive at a total score. The internal consistency $($ alpha $=0.94)$ and inter-observer reliability $($ ICC $=0.98)$ of the ADL-observations are good. ${ }^{13}$

\section{Additional measures}

In order to assess whether the patients with apraxia suffered other neuropsychological deficits as well, the following measurements were conducted.

\section{Comprehension of language}

An aphasia test battery for auditive and verbal use of language has been developed and validated in the Netherlands (SAN-test). ${ }^{14,15}$ We use the subtest 'comprehension of sentences' designed to test the ability to comprehend simple sentences. A maximum score of 45 was given when all sentences were judged correctly by the patient.

\section{Cognitive orientation}

The Cognitive screening test (CST) is a short, reliable, and valid standardized instrument to measure cognitive impairments, specifically due to dementia, by asking the patient about simple facts, usually learned in the past. The test differentiates very well between healthy elderly and patients with the dementia syndrome. ${ }^{16}$ When all items of the test are answered correctly, the patient gets a score of 20.

\section{Unilateral visual neglect}

The Star cancellation test is a subtest of the Behavioural inattention test. ${ }^{17}$ It is a short test to measure unilateral visual neglect and is considered to probably be the most sensitive single test. ${ }^{2}{ }^{28}$ The patient has to detect a specific stimulus in a visual array of many different stimuli. The number of target stimuli (i.e. small stars) that are omitted is scored ; the maximum number of omissions is 56.

\section{Short term memory (digit span)}

A series of digits was presented verbally to the patients. Patients were asked to recall each series immediately upon presentation (forward recall). The total score represents the number of series remembered correctly. ${ }^{19}$

\section{Testing procedure}

Patients with apraxia as well as the control patients without apraxia were tested by the experimenter in a one-hour testing session, during which the apraxia test, the motor functioning test and the tests for additional impairments were administered. The patients sat facing the experimenter in a quiet room suitable for testing. The occupational therapists carried out measurements assessing the level of disabilities in ADL-activities. The ADL observations were conducted in an environment appropriate 
Heugten, C.M. van, Dekker, J., Deelman, B.G., Stehmann-Saris, J.C., Kinebanian, A.

Rehabilitation of stroke patients with apraxia: the role of additional cognitive and

motor impairments.

Disability and Rehabilitation: 22, 2000, nr. 12, p. 547-554

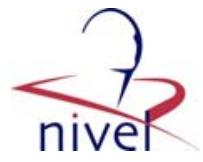

for the task at hand, as well as at a time of day relevant for the specific task (e.g. washing in the bathroom, after getting out of bed).

\section{Statistical analyses}

The baseline scores of the apractic patients were compared to normscores to determine whether they suffered from additional impairments. Next, the scores in the present sample were compared to a control group of stroke patients without apraxia to determine whether the presence of additional impairments was related to the presence of apraxia. The scores of the two groups were compared using t-test for independent samples.

Change scores, indicating improvement in functioning, were calculated for each patient by subtracting the results after twelve weeks follow-up from those at baseline. The t-test for paired samples was used for comparison of measures before and after treatment. Effect sizes were calculated by dividing the mean changes in scores by the standard deviation of the pre-test score. The analyses as regards treatment outcome are presented in more detail in Van Heugten et al. ${ }^{20}$

Correlation coefficients between the pre-test scores (baseline performance, additional neuropsychological tests, and some patient characteristics) and the change score of the main outcome measure (i.e. the ADL-observations) were calculated. Bivariate relationships are expressed by means of Pearson's product moment correlation coefficients. In addition, for categorical variables Eta is presented. Eta was used for the relationships between the ADL-change score and gender (male, female), type of stroke (cerebral haemorrhage, cerebral infarction) and location of treatment (hospital, rehabilitation centre, nursing home). Eta varies between 0 and 1 and is used for associations between nominal and interval variables. ${ }^{21}$

All statistical analyses were performed using SPSS/ PC+, version 5.0. The significance level was set at 0.05 .

\section{RESULTS}

\section{Characteristics of the patients}

A total of 45 patients with apraxia were included in the study. For 33 stroke patients with apraxia pre- and posttreatment scores were available; twelve patients were excluded from the measurements at 12 weeks for various reasons (e.g. bad medical condition, refusal of treatment, death and early discharge).

Thirty-sex patients without apraxia constituted the control group. This group of patients was only tested at baseline. The two groups did not differ significantly as regards gender, type of stroke and time since stroke ; the apraxia patients, however, were significantly older than the stroke patients without apraxia $(\mathrm{p}<0.01)$. In table 1 the characteristics of the patients with and without apraxia are presented.

\section{[ TABLE 1 ]}

\section{Baseline scores}

The baseline scores of the patients with and without apraxia are shown in table 2. As expected, the baseline score on the apraxia test was much lower for the patients with apraxia than for the patients without apraxia $(\mathrm{t}=6.16 ; \mathrm{p}<0.01)$. On the motor functioning test the mean score of the patients with apraxia was not significantly different from the reference group without apraxia. The scores suggest that both the patients with apraxia and the patients without apraxia suffered from primary motor impairments.

\section{[ TABLE 2 ]}

The poorest performance on the test for comprehension of language by healthy persons older than 65 years (normscore) is 40 items correct. ${ }^{14}$ Seventy three per cent of the apractic patients in our group scored below this normscore, and only one patient reached the maximum value of 45 . In the group 
Heugten, C.M. van, Dekker, J., Deelman, B.G., Stehmann-Saris, J.C., Kinebanian, A.

Rehabilitation of stroke patients with apraxia: the role of additional cognitive and

motor impairments.

Disability and Rehabilitation: 22, 2000, nr. 12, p. 547-554

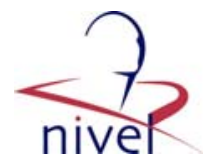

without apraxia $9 \%$ of the patients scored below the normvalue, while more than half of the subjects reached the maximum score. The mean scores of the two groups differed significantly $(t=6.83 ; \mathrm{p}<$ 0.01). On the Cognitive screening test a maximum of 20 could be reached, while the patients with apraxia in our study obtained a mean score of 13.8. Normscores indicating the presence of dementia are based on a cut-off score of $12.1 ;{ }^{16} 41 \%$ of the apractic patients obtained a score below this value. In the control group only $6 \%$ scored below the normscore. The two groups differed significantly $(\mathrm{t}=$ 4.26; $\mathrm{p}<0.01$ ). If more than 3 stimuli are omitted on the test of unilateral visual neglect the subject is considered to show some degree of neglect. This normscore is based on a study by de Kort ${ }^{22}$ in which the lowest score of subjects in a control group (i.e. elderly persons without central neurological damage) was the omission of 2 stimuli. In the group of patients with apraxia $19 \%$ of the patients had omitted more than 3 stimuli, while in the group of patients without apraxia $6 \%$ of the patients had more than 3 omissions. The groups did not differ significantly. The last test was included to measure short term memory functioning. Apractic patients scored significantly worse than non-apractic patients $(\mathrm{t}=5.64 ; \mathrm{p}<0.01)$.

In summary, the presence of apraxia seems to be associated with impairments in motor functioning, comprehension of sentences, cognitive orientation and short term memory functioning.

\section{Treatment outcome}

Treatment outcome is presented in more detail in van Heugten et al. ${ }^{20}$ The results are summarized in table 3; the baseline scores, 12 week results and effect sizes are presented. The ADL observations showed large improvement ; apraxia and motor functioning showed significant though less improvement. As was expected, the effect sizes for apraxia and motor functioning were small compared to the effect size for the ADL-observations.

\section{[ TABLE 3 ]}

\section{Bivariate relationships}

In table 4 the correlations between all baseline scores and improvement in independent functioning (i.e. the change scores of the ADL observations) are presented. A significant correlation was found between the level of ADL functioning at baseline and the improvement in ADL functioning, as measured with the ADL observations $(r=0.69 ; \mathrm{p}<0.01)$. This means that the more dependent the patient is before treatment, the more improvement is seen after treatment. The improvement in ADL functioning is negatively associated with the level of apraxia and motor functioning at baseline $(\mathrm{r}=$ $0.46, p=0.02$ and $r=-0.42, p=0.04$ respectively). For both tests, it applies that the lower the pre-test score (i.e. the more severely impaired the patient) the more improvement in ADL functioning was seen. In other words, the most severely impaired patients showed the most marked improvement. The significant relationships (ADL-improvement and baseline ADL functioning, motor functioning and apraxia) are graphically presented in figure 1 .

\section{[ TABLE 4 ]}

\section{[ FIGURE 1 ]}

Other neuropsychological impairments at baseline did not show significant correlations with the change score of the ADL observations. As for age, time since stroke, gender, type of stroke and treatment setting no significant correlations were found with the change in ADL functioning (all pvalues exceeded 0.10 ).

\section{DISCUSSION}

The aim of the present study was twofold. First, it investigated which cognitive and motor impairments are associated with apraxia following stroke. And second, prognostic variables were examined related to functional outcome. 
Heugten, C.M. van, Dekker, J., Deelman, B.G., Stehmann-Saris, J.C., Kinebanian, A.

Rehabilitation of stroke patients with apraxia: the role of additional cognitive and

motor impairments.

Disability and Rehabilitation: 22, 2000, nr. 12, p. 547-554

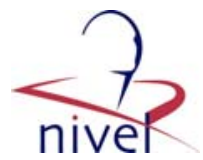

The presence of additional impairments in stroke patients was found to be far more prominent in the group of patients with apraxia than in the group of patients without apraxia. In comparison with patients without apraxia, the apractic patients in our sample seemed to have more problems in comprehending sentences, in cognitive dementia like impairments, and in remembering series of digits correctly. Motor impairments were present in both groups.

Surprisingly, this cognitive comorbidity did not seem to cause a less favourable treatment outcome. Apparently, the presence of additional cognitive impairments was not an impediment for this kind of treatment. Likewise, bad motor functioning was expected to have a negative influence on functional improvement as well. ${ }^{3}$ However, more improvement in ADL functioning was found in those patients who initially had more problems in motor functioning. Next we investigated the predictive value of baseline performance on the apraxia test and the ADL observations. In our study it appeared that for both instruments the initially more severely impaired patients showed the most marked improvement in independent functioning.

This latter result was remarkable and not expected ; further investigation of the measurements is therefore needed. Inspection of figure 1 provided insight. For the relationship between the ADL baseline scores and the improvements in ADL functioning (figure 1a) the regression to the mean phenomenon should be considered : patients starting with a positive score will tend to get a lower score, while patients with a negative score at the start will tend to get a better score. This statistical problem cannot be seen in the figure. It can, however be seen in figure $1 \mathrm{~b}$ that part of the data is clustered in the upper corner. This indicates a ceiling effect : patients functioning independently before treatment cannot improve any more. This could indicate that the ADL observations are not capable of detecting changes towards the positive end of the scale. Figure $1 \mathrm{c}$ and $1 \mathrm{~d}$ do not provide insight into this matter, because the points in the graph are scattered more (due to a lower $r$ and probably due to a less linear relationship).

As regards the ADL observations, the observed ceiling effect raises an issue of discussion. In the present sample a considerable number of patients already functioned rather independently before treatment and thus could not improve any more ; this is a natural ceiling effect. However, on the basis of clinical decision making the patients functioning good before treatment were admitted to the treatment program. If these patients could not improve any further this would not have been decided. Thus, it can also be argued that the ADL observations were not capable of detecting clinically relevant improvements in independent functioning ; this would suggest an instrumental ceiling effect. Not all activities which were observed might have been sufficiently difficult to raise problems in functioning. A more complex task could have shown a stronger differentiation in improvement towards the end of the scale.

As regards the prognostic value of patient characteristics the following conclusion can be drawn. We did not find age to be a significant predictor. The other demographic variables we examined did not yield significant results either. Unfortunately, in our study we could not examine the relationship between functional outcome and the site of the lesion, which appeared to be a relevant factor in the recovery of ideomotor apraxia. ${ }^{4}$

In conclusion, the bivariate relationship were presented with some words of caution ; more definite conclusions concerning the regression to the mean effect and the ceiling effect should be drawn on the basis of additional studies. There seems to be a need for an instrument differentiating more towards the positive end of the scale. We conclude that there was no indication that the effect of this training is weaker in more severely disabled patients. Neither the presence of additional cognitive impairments nor the severity of motor deficits nor old age seemed to be an indication for refraining from treating apraxia. 
Heugten, C.M. van, Dekker, J., Deelman, B.G., Stehmann-Saris, J.C., Kinebanian, A. Rehabilitation of stroke patients with apraxia: the role of additional cognitive and

motor impairments.

Disability and Rehabilitation: 22, 2000, nr. 12, p. 547-554

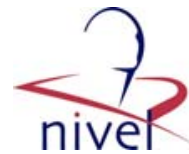

\section{TABLES AND FIGURES}

Table 1 Characteristics of the stroke patients with apraxia and the control patients without apraxia

\begin{tabular}{lcc}
\hline & $\begin{array}{l}\text { patients } \\
\text { with apraxia }\end{array}$ & $\begin{array}{l}\text { patients } \\
\text { without apraxia }\end{array}$ \\
\hline Gender & 18 & 14 \\
Male & 15 & 22 \\
Female & & \\
Age & 70.1 & 59.9 \\
mean & 11.0 & 12.8 \\
sd & $39-91$ & $35-88$ \\
range & & \\
Location of treatment & 11 & 10 \\
Hospital & 14 & 20 \\
Rehabilitation centre & 8 & 6 \\
Nursing home & & \\
Type of CVA & 9 & 11 \\
Cerebral haemorrhage & 23 & 23 \\
Cerebral infarction & 1 & 13.5 \\
Unknown & & 15.8 \\
Time since CVA (in weeks) & 8.9 & 36 \\
mean & 5.2 & \\
sd & 33 & \\
$N$ (total) & & \\
\hline
\end{tabular}

Table 4 Bivariate relationships between change scores of the ADL observations and baseline scores neuropsychological scores and patien characteristics

\begin{tabular}{lc}
\hline Change scores & ADL observations \\
\hline Baseline scores & $-0.46^{*}$ \\
Apraxia & $-0.42^{*}$ \\
Motor functioning & $0.69^{* *}$ \\
ADL observations & \\
Neuropsychological functioning & -0.12 \\
Comprehension of language & -0.09 \\
Cognitive orientation & -0.02 \\
Neglect & -0.22 \\
Short term memory & \\
Patient characteristics & -0.03 \\
Age & -0.01 \\
Time since stroke & 0.05 \\
Gender & 0.34 \\
Type of stroke & \\
Location of treatment &
\end{tabular}

$\wedge$ Eta.

$* * \mathrm{p}<0.01 ; * \mathrm{p}<0.05$

Table 2 Baseline measurements for patients with apraxia and patients without apraxia

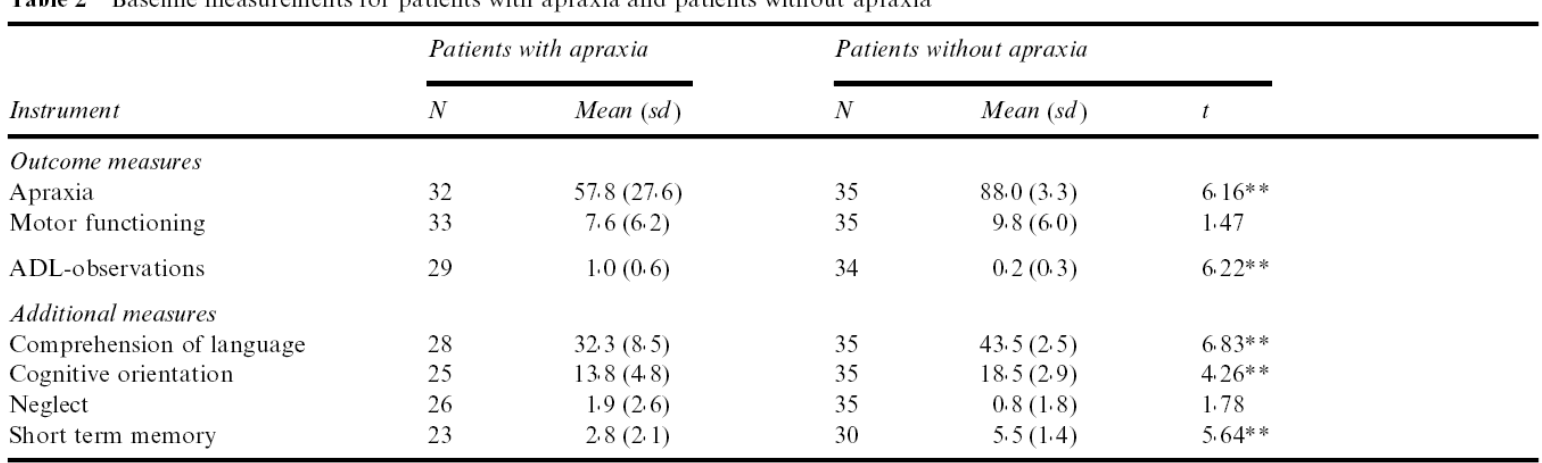

$* * \mathrm{p}<0.01 ; * \mathrm{p}<0.05$.

Table 3 Treatment outcome

\begin{tabular}{lcccl}
\hline Instrument & $N$ & Baseline & Post-treatment & Effect size \\
\hline Apraxia & 31 & $58.10(28.0)$ & $67.58(26.9)^{* *}$ & 0.34 \\
Motor functioning & 33 & $7.64(6.2)$ & $8.80(6.3)^{*}$ & 0.19 \\
ADL-observations & 25 & $0.98(0.65)$ & $0.38(0.47)^{* *}$ & 0.92 \\
\hline
\end{tabular}

$* * \mathrm{p}<0.01 ; * \mathrm{p}<0.05$. 
Heugten, C.M. van, Dekker, J., Deelman, B.G., Stehmann-Saris, J.C., Kinebanian, A. Rehabilitation of stroke patients with apraxia: the role of additional cognitive and motor impairments.

Disability and Rehabilitation: 22, 2000, nr. 12, p. 547-554
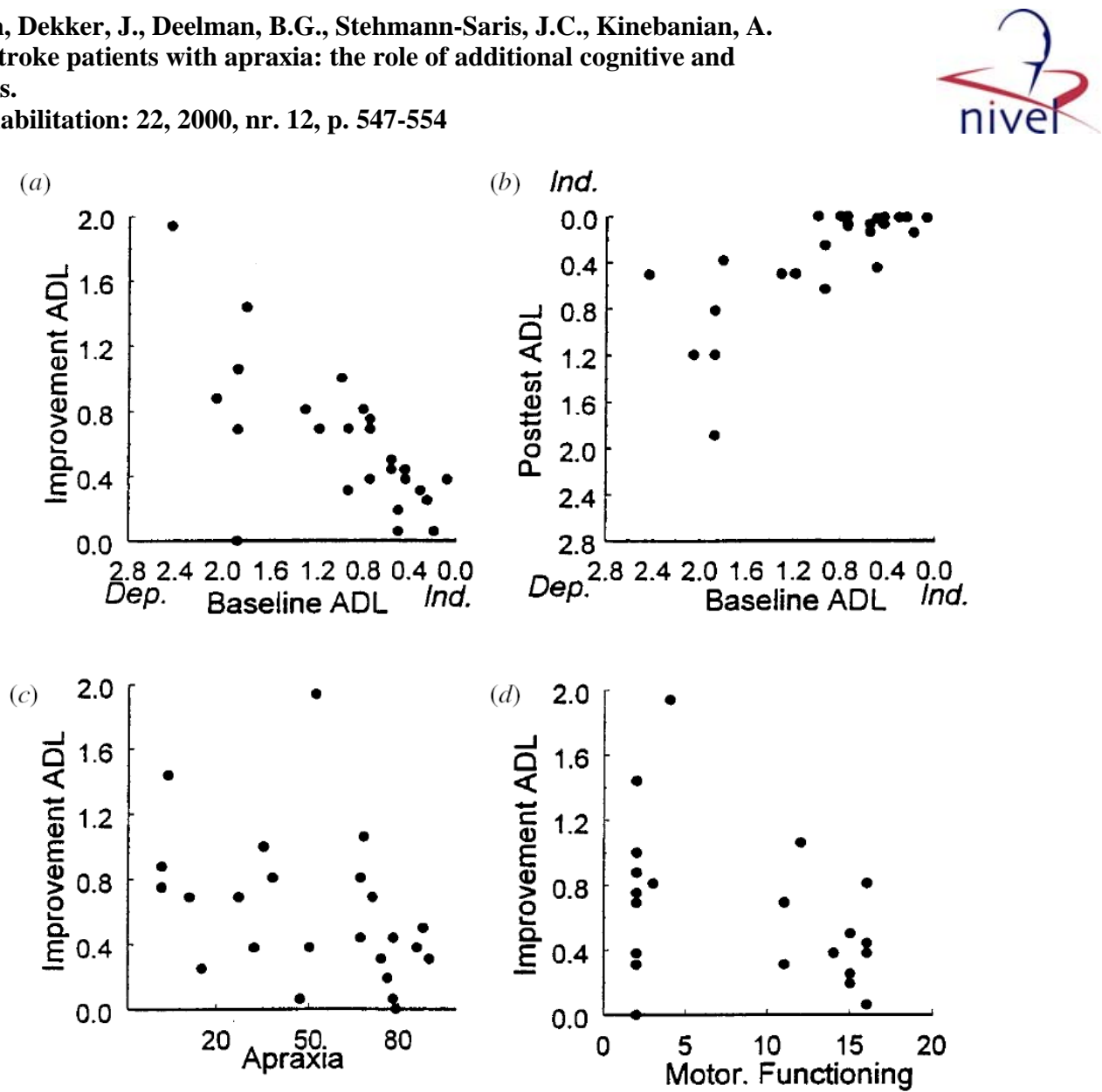

Figure 1 Relationships between the change score of the ADL-observations and the baseline score of the ADL-observations (1a), the baseline ADL versus posttest ADL scores (1b), the apraxia test (1c), and the motor functioning test (1d).

\section{REFERENCES}

1. Jongbloed L. Prediction of function after stroke : a critical review. Stroke 1986; 17: 765-776.

2. Graham JRF, Harwood DMJ, Barer DH. Predicting the outcome of acute stroke: prospective evaluation of five multivariate models and comparison with single methods. Journal of Neurology, Neurosurgery and Psychiatry 1992; 55: 347-351.

3. Kwakkel G, Wagenaar RC, Kollen BJ, Lankhorst GJ. Predicting disability in stroke : a critical review of the literature. Age and ageing 1996; 25: 479-489.

4. Basso A, Capitani E, Della Sala S, Laiacona M, Spinnler H. Recovery from ideomotor apraxia.A study on acute stroke patients. Brain 1987; 110: 747-760.

5. Sundet K, Finset A, Reinvang I. Neuropsychological predictors in stroke rehabilitation. Journal of Clinical and Experimental Neuropsychology 1988; 10: 363-379.

6. World Health Organization. Special report. Recommendations on stroke prevention, diagnosis and therapy. Stroke 1989; 20: 1407-1431.

7. Crevel $\mathrm{H}$ van. Consensus cerebro vasculair accident. Nederlands Tijdschrift voor Geneeskunde 1991; 135: 2280-2288.

8. Kolb B, Whishaw IQ. Fundamentals of Human Neuropsychology (3rd edition). New York: Freeman, 1990.

9. Stehmann-Saris JC, Heugten CM van, Kinebanian A, Dekker J. Ergotherapie Protocol Voor Diangostiek en Behandeling van Apraxie Bij CVA-Patienten. Utrecht: NIVEL; Amsterdam: HVA, 1996.

10. Heugten CM van, Dekker J, Deelamn BG, Stehmann-Saris JC, Kinebanian A. A diagnostic test for apraxia in stroke patients: internal consistency and diagnostic value. The Clinical Neuropsychologist 1999; 13: 1-11.

11. De Renzi E. Apraxia. In : Boller F and Grafman J (eds) Handbook of Neuropsychology, volume 2. Amsterdam: Elsevier, 1989.

12. Wade DT. Measurement in Neurological Rehabilitation. Oxford: Oxford University Press, 1992.

13. Heugten CM van, Dekker J, Deelman BG, Stehmann-Saris JC, Kinebanian A. Assessment of disabilities in stroke patients with apraxia: internal consistency and inter-observer reliability. The Occupational Therapy Journal of Research 1999; 19, 1-19. 
Heugten, C.M. van, Dekker, J., Deelman, B.G., Stehmann-Saris, J.C., Kinebanian, A. Rehabilitation of stroke patients with apraxia: the role of additional cognitive and motor impairments.

Disability and Rehabilitation: 22, 2000, nr. 12, p. 547-554

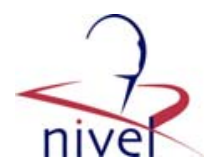

14. Deelman BG, Koning-Haanstra M, Liebrand WBG, Burg W van den. De SAN-test: Eenlisse : Afasietest Voor Auditief en Mondeling Taalgebruik, Handleiding. Swets and Zeitlinger, 1981, 1987.

15. Visser RSH, Vliet-Mulder JC van, Evers A, Laak J ter. Documentatie van Tests en TestreseArch in Nederland. Nederlands Instituut voor Psychologen. Nijmegen: van Manneren, 1982.

16. Graaf A de, Deelman BG. De Cognitive Screening Test. Lise : Swets and Zeitlinger, 1991.

17. Halligan PW, Cockburn J, Wilson BA. The behavioural assessment of visual neglect. Neuropsychological Rehabilitation 1991; 1: 5-32.

18. Halligan PW, Marshall JC, Wade DT. Visuospatial neglect: underlying factors and test sensitivity. Lancet 1989; 2: 908-910.

19. Lezak, MD. Neuropsychological Assessment (3rd edition). New York/Oxford: Oxford University Press, 1995.

20. HeugtenCM van,Dekker J, Deelman BG, Dijk AJ van, Stehmann- Saris JC, Kinebanian A. Outcome of strategy-training in stroke patients with apraxia: a phase-II study. Clinical Rehabilitation 1998; 12: 294-303.

21. Ferguson, GA. Statistical Analysis in Psychology and Education. London: McGraw-Hill, 1966, 246-249.

22. Kort de PLM. Neglect. Dissertation. Groningen: University of Groningen, 1996. 